\title{
Genetic Algorithm Dependent Qos Aware Adaptive Subcarrier Allocation Scheme in Cognitive Radio Networks
}

\author{
Rajalakshmi, P.Sumathy
}

\begin{abstract}
Cognitive Radio Network technology is an efficient and intelligent system with the intention of detecting automatically about the availability of channels in a wireless spectrum and changing transmission parameters to facilitate more communication.In Quality of Service aware spectrum decision making system is locating the spectrum bands all the way through in view of the application necessities by means of the self-motivated nature of the spectrum bands.Here, an innovative adaptive sub-carrier allocation mechanism is proposed for Cognitive Radio Networks by rearranging the functional parameters anchored in the Proportional Resource Algorithm and Genetic Algorithm.This proposed scheme is designed to achieve a divergent performance standard for subcarrier allocation and power allocation system of the Cognitive Radio Network users. The suggested scheme is proven to be effective by maximizing the allocation of data rate for each subcarrier. Moreover, by increasing the overall network throughput, the numbers of satisfied users within bandwidth limits are maintained with the quality of service. In fact, it locates the maximum channel availability.
\end{abstract}

Keywords: Cognitive Radio Technology, Genetic Algorithm, Quality of Service,Proportional Resource Algorithm, Subcarrier Allocation

\section{INTRODUCTION}

The Cognitive Radio Technology is a wireless conceptual communication system which is sensitive to its surrounding environment. In general, it can be expected to pay attention to the parameters such as channel availability, accessible channels, availability of channels and the nature of data to be transmitted in the forms of modulation which could be used.Cognitive Radio Technology is one of the new longstanding developments going to take place along with the communication systems. After the Software Defined Radio (SDR), which is gradually becoming more in reality becomes the next major step forward by the Cognitive Radio Technology, allowing the system to develop in an efficient way. Cognitive Radio Technology applies a variety of mechanisms which includes Adaptive Radio and Software Defined Radio (SDR) where hardware components have been replaced with intelligent software.

Revised Manuscript Received on April 25, 2020.

*Corresponding Author

Rajalakshmi*, Scholar, Department of Computer Science, Bharathidasan University, Trichy, Tamil Nadu, India - 620023, laks2020cs@gmail.com

Dr. P. Sumathy, Assisatnt Professor, Department of Computer Science, Bharathidasan University, Trichy, Tamil Nadu, India - 620023, sumathy.p@bdu.ac.in

(c) The Authors. Published by Blue Eyes Intelligence Engineering and Sciences Publication (BEIESP). This is an open access article under the CC BY-NC-ND license (http://creativecommons.org/licenses/by-nc-nd/4.0/)
In the present communication scenario, Radio Frequency (RF) technology plays a vital role in many aspects of electronics field such as Wi-Fi, WiMAX, NFC, RFID, and many other devices.This technology is known as Cognitive Radio where its main benefit lies by enabling an opportunity to access an additional bandwidth for wireless devices in order to maximize their throughput. Mitola. J, et al,Suggested that the hopeful method in the cognitive radio model, the spectrum consumption is attached using spectrum chance. An innovative research area envisages magnifying Cognitive Radio Technology to improve bandwidth capacity through assisting fresh wireless users [1].

In modern communication structure, the wireless frequency bandwidth has become recognizable public services. The progression of Frequency band control and determined spectrum allocation are waging a dispute with the spectrum allocation scheme offered based on high demand.The distribution strategies are derived from the static model where the frequency allotment is sought by government agencies around the world, such as the Federal Communication Commission (FCC). This system directs the utilization of the valuable frequency resources which are accessible by the spectrum. The Channel selection practice is playing a significant role to facilitate the cognitive radio along the way to collect the strongest developed channel enclosed by the gathering of recognized channels. The Cognitive Radio Technology should dynamically regulate the parameters of the physical layer, such as frequency, bandwidth, power, etc. After the channel has been allocated to the cognitive radio system, while, if the Primary User arrives, the Cognitive Radio system must suspend its operation and re-allocate the system for an uninterrupted service which steams QoS. The frequent transmission cycle suspensions damage the QoS in the cognitive radio system. Though these works show the QoS requirements, there is still insufficient in the works. Farah.J et al, Tendered the channel allocating channels and resources with the QoS supported to achieve the goal with maximum data rates for all users in cognitive networks, even though this algorithm is highly complex in nature [2].

Amir Ghasemi et al,Have mentioned that numerous causes based on uncertainty which involve channel, noise, sensing interference limit and so on in the Cognitive Radio system. These issues are solved by the usage of the bandwidth in the Cognitive Radio System. Yang L, et al, Have presented an innovative channel access scheme in order to overcome disruptive circumstance in cognitive radio network. And to concentrate the complex factors, that

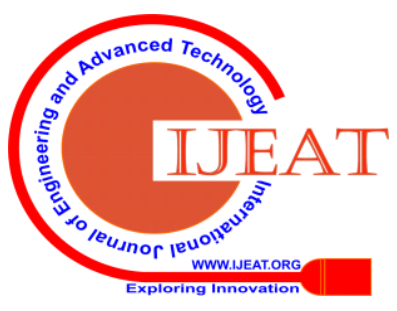


emphasizes how to build a network based on the wireless Cognitive Radio System. The main aim is to provide a pioneering view of the wireless communication network and resource allocation issues during the development of the channel selection scheme.

Akyildiz.I.F,Has proposed the system intended for cooperative bandwidth sensing in Cognitive Radio Technology. This method realizes communication management functions that are required for Cognitive Radio Technology by means of targeted, efficient to meet challenges such as avoidance of intrusion, knowledge of QoS and seamless communication. Cognitive Radio Network specifically monitors the internal and external system environment. This system uses the environment approach and modifies its internal states for statistical variations in incoming Radio Frequency stimuli. Such modifications are known by its functionalities relevant to complex spectrum management. The two key distinctive features of CR defined to resolve DSM roles are cognitive capacity and reconfiguration [3].

Optimization is the process accompanying the given conditions to achieve the most outstanding result. All such decisions provides either to minimize effort or to maximize profit. The gain will usually be expressed as a function of some variables. Therefore, optimization is the process of finding the conditions which give a function's maximum or minimum value. When optimizing a design, the design objective might be simply to reduce production costs or optimize production efficiency. The optimization algorithm is a procedure that is done iteratively by comparing different solutions until an optimal or acceptable solution has been found. In addition to the object of the linear programming which has many limitations and these limitations is considered to be the rare linear functions of the optimization parameters. Consequently paradigm will shift complex problems for more simplified methods of programming. Genetic Algorithm plays a very important role in the quest of modification and optimization techniques in order to find approximate optimal solutions due to its multi-objective CRN optimization capability [4].

The Genetic Algorithm is put into effect for streamlining the multi-carrier transmitters and single specified software defined radio applications. Different network channel selection strategies will be used as the fundamental genes to adapt operating parameters such as bandwidth, power consumption and bit error rate (BER). The objective function comes together with an optimal value where the termination condition is arriving at relating to desired criteria. Gardner.W et al, Have suggested an optimization scheme is to minimize the BER, and to maximize the power consumption for efficient throughput by means of overflowing the comprehensive parameter by the reconfiguration simulation model using the comparable GA parameters to ensure the QoS. However, the solution to accommodate the effects of the PU operation on the CRN output is not addressed to all of the schemes mentioned [5].

\section{LITERATURE SURVEY}

Cognitive Radio Technology is considered to be a prototype for wireless communication system where the transmission parameters are altered by a wireless communication node to communicate proficiently and avoid interference with users. In cognitive radio systems, the method of predicting the near future availability of the primary user (PU) channel state (i.e., spectrum occupancy) is relatively crucial to prevent interference with its transmission by a cognitive radio system user (i.e., secondary consumer (SU)).

The bandwidth accessibility mechanisms in a cognitive broadcasting network allow an unlicensed consumer to dynamically adjust their operating frequency based on the conditions of the environment.Address these issues in the following ways.

The spectrum mobility functions in a cognitive radio network allow an unlicensed user to change its operating spectrum dynamically based on the spectrum conditions. This issue can be addressed in following ways.

- Looking for the best frequency range:

- Protocol stacks adaptation:

- $\quad$ Self-coexistence and synchronization.

Nguyen.V.T. et al stated about the fundamental concepts of the Cognitive Radio Networks through subsequent of three key points.

- $\quad$ To acquire the intelligence based on its operations with maintenance.

- $\quad$ Based on the operational parameters and procedures tha $\mathrm{t}$ are dynamic and cooperatively adjust to satisfy the set of predetermined strategies.

- $\quad$ Benefit from the outcomes achieved.

When data overhead enhances a large sensory data because of the cognitive radio networks are be capable of sensing bandwidth and able to scan a wide range of frequency. Consequentially the huge amount of the data is incompetent.

Nevertheless considerately sensing data process the bundle of bandwidth and the same will be approved without gaining a lot of overhead since only approximate sensing information is needed, eradicating require signal processing schemes at the receiver side will drop the data load. Furthermore, the broadband channel has to be scanned. At the time of updating a portion of it is altered which is merely the altered information and not all the information of the entire scanned spectrum. Wang. $\mathrm{P}$ et al. suggested that the GLRT sensing device observed the signal structure which deletes the noise variance as an unknown criterion. Even then it is examined by the ML parameter evaluation which underlies the GLRT scheme [6].

A study concerning spectrum sensing techniques was performed by MansiSubhedar et al, where the problems and their related issues involved in accomplishing the spectrum sensing techniques are explored in a detailed manner and presents the various comparative schemes of analysis.

While considering the challenges presented by Cognitive Radio Technology, the use of the spectrum sensing method begins to appear as a crucial requirement to achieve satisfactory results in terms of efficient use of the available spectrum and limited interference with licensed primary users. The design of the cognitive radio network, as defined in this paper, involves the participation and interaction of many advanced techniques including distributed spectrum sensing, interference management, cognitive radio reconfiguration management and cooperative communications 
The method used to detect interference of spectrum sensing should be accurate with time, So that the primary user does not endure to use their licensed bandwidth from the CR system.

GoutamGhosh et al, have reviewed plentiful dissimilar dynamic spectrum access methods and spotlighted that the game theoretic method is a significant. This method is used for spectrum sharing at large quantities and gives attention to the spectrum management in the Cognitive Radio Technology. Majority of people are accepting the Game theory technique to achieve the equilibrium solution to the issue of the spectrum sharing. Ahmed Mohammed Mikaeil has offered the spectrum occupancy prediction model for a single user cognitive radio network utilizing the Bayesian online learning model. In this model, the detection sequence of primary user channel state availability is represented as a time series changing over the time between two states namely PU idle and PU occupied. The Bayesian online learning system is employed to forecast the changes in the states of the time series in advance.

As a final point, the performance of the proposed algorithm utilizing a simulated PU detection sequence for estimation. The observed simulation outcomes have proved the efficacy of the BOL model in foretelling PU channel state availability. RayanAbdelazeemHabboubSuliman et al have accomplished a survey, on the use of bandwidth in cognitive radio networks. The use of dynamic spectrum is used to optimize spectrum efficiency, reduce costs, and increase the number of wireless users who develop more spectrum characteristics in advance. Hassaan Bin Ahmad has recommend spectrum sensing plan for Cognitive Radio Technology by utilizing Ensemble Classifier. With the aid of machine learning technique and cyclostationary functionality, the discovery of spectrum sensing is processed to overcome Cognitive Radio Network problems. Restrictions on low signal-to-noise ratio (SNR), location threshold detection, and computational costs are addressed by proposing a classifier for the ensemble. A dataset that contains various orthogonal frequency-division multiplexing signals at different SNRs is generated primarily, cyclostationary features are subsequently extracted using FFT accumulation process. Eventually, with the aid of the extracted features, the proposed ensemble classifier was trained to sense the Primary User's signal under low SNR conditions. Genetic algorithms are well adapted for optimizing the issues which involves larger in search spaces. Thomas W. Rondeau et al. suggests the adaptive component for the genetic algorithm which simulations for complete a chromosome-defined device. The genes of both the chromosome symbolize the modifiable parameters within a given machine and by modifying the chromosomes genetically. The Genetic algorithms is used to locate a selection of parameters for tuning the radio to the current users needs[7]. Bruce.A.Fette has classified the spectrum sensing techniques into two namely; direct method and indirect method. The direct method can be seen as an approach to the frequency domain, where the measurement is carried out directly from the signal. The calculation is done in the indirect method or time domain approach, based on the signal's auto correlation. Another way to categorize the methods of spectrum sensing and estimation is by making a group into a parametric method based on a model and a non-parametric method based on a perioddogram.
Using non-dominated sorting to optimize CR parameters, Guo-ying Yang et al. recommend a multi-objective genetic algorithm in DNA. To converge to the global Paretooptimal line, non-dominated sorting and clone operator are employed. The simulation results show that DNA-GA is capable of developing the search capability by leading genetic manipulation and achieving excellent population diversity where DNA-GA is feasible for validating the Cognitive Radio Parameter for Optimization [8]. Si Chen et al, through the genetic algorithm, indicated that the optimization systems as well as several assisting mechanisms in authentic network achievements make these systems more efficient and reliable. The cognitive radio network is capable of sensing the existing environmental conditions and modifies its operating parameters automatically which enhances the performance. The suggested techniques will optimize individual wireless devices within the network from their adjacent devices, using partial operating parameters and environmental information. The proposed method uses multiplexing of the non-contiguous orthogonal frequency division (NCOFDM) to all wireless connections which can acquire a Dynamic Spectrum Access (DSA) system. The proposed method participates in minimizing the level of bit error and minimizing the out- of-band.

S.Chen et al. suggested various approaches to improve the convergence time and to maximize the performance of a genetic algorithm. It is recalled that the genetic algorithm achieves a well-managed solution to the system's complexity. Beibei Wang et al. have conducted a survey regarding the progression of cognitive radio networks. The cognitive radio is capable of theoretically using any bandwidth gap and conducts a scan across a wide range of spectrum, resulting that the large amounts of data which becomes inefficient in terms of data throughput, delay response requirements and energy usage. It dramatically increases the workload of data, The collaborative sensing data presents many challenges, it could be carried out without incurring much workload because only approximate sensing information is needed, eliminating the need for complex signal processing schemes in the receiver and reducing data load.

SaleemAslam et al. suggested a Dynamic Channel Selection and Parameter Adaptation (CSPA) approach that is based on genetic algorithms to improve he QoS of Cognitive Radio network by selecting the best channel for performance control and Primary $U$ requirements. The CSPA tackles the channel switching problem and enables better QoS for Cognitive Radio network customers. The simulation results indicate that CSPA works well [9]. Yanchao Yang et al. developed a cognitive engine to engage the dynamic wireless environment and their demands through the combination of Genetic Algorithm and Radial Basis Function Neural Network. This method connects decision-making table created by pre-processing perceptive information in order to train the learning model Radial Basis Function (RBF). GA is used to alter Radial Basis Function calculates the selected appropriate fitness solution. The program is subsequently trained where the performance of two benchmark functions is measured.

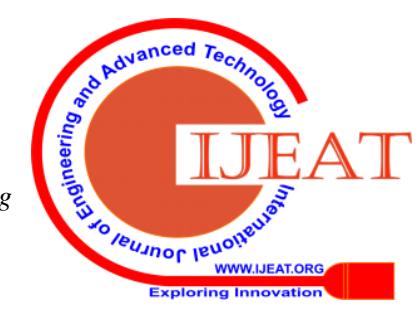


The simulated results show the effectiveness approach and the cognitive model suggested which are efficiently attaining the cognitive radio goal for learning and reconfiguration.

Jamal Elhachmi et al. have proposed the cognitive radio spectrum allocation scheme that includes the problem formulation and employ through genetic algorithm for channel allocation. The system provides a competent way to access the available bandwidth for both primary and secondary users. With the support of modern sophisticated crossover and mutation operators of genetic algorithms which recommended the scheme to designed in such way to ensure the validity of channels and speedy convergence. The simulation responses show that the proposed scheme achieves reasonable results with reduced network interference which efficiently improves the spectrum efficiency as compared to the existing methods[10]. Chunmun Singh et al. suggested model includes in collaborative situation and effective spectrum sensing procedure for cognitive radio network by means of soft computing techniques. The suggested model includes in collaborative situation and effective spectrum sensing procedure for cognitive radio network by means of soft computing techniques. The QoS parameter is considered under these works for the bit error rate, band power, and data rate of the secondary users. The GA optimizes antenna selection matrix parameters, transmitter power, modulation type, modulation order, pulse shaping filter roll-off factor, and symbol rate to achieve QoS.

\section{PROPOSED SUBCARRIER ALLOCATION SCHEME IN CRN WITH PARAMETER RECONFIGURATION THROUGH GENETIC ALGORITHM}

In this proposed scheme, the resource allotment algorithm and genetic algorithm are exploited in order to amend the operating frequency parameters. The multi objective optimization problem through equality and inequality constraint is utilized. The proposed subcarrier allocation scheme is devised with help of cognitive radio network as rendered in figure 2 . The number of CR users are embodied from $n=1,2,3 \ldots$..All Cognitive Radio netrwork users are periodically sensing their environment and collecting information about the Primary User's working under Base Station of the Primary user. Here, the Generalized Likelihood Ratio Test (GLRT) is considered more for spectrum sensing that uses the Eigen Values pertaining to the noise subspace of the signal vector sample covariance matrix obtained. For large numbers of sensors, there is a greater likelihood of detection in unknown parameters and no knowledge of loudness, or signal power is required. The proposed scheme initially takes into account the existence of reliability of PU transmitters which is a significant constraint since the network in question operates with multiple PU networks. The proposed scheme's prime objective is to adjust Fast RF parameter.The GLRT sensing scheme is used for the intrinsic signal structure and views the noise variation as an uncertain parameter, in line with Wang. P et al. The common structure of the GLRT is mentioned for the problem is as below

The general form is given as

$$
T_{G L R T}=\frac{\max _{h, \sigma_{s}^{2}} P\left(X \mid H_{1}, h, \sigma_{s}^{2}\right)}{\max _{\sigma_{s}^{2}} P\left(X \mid H_{0}, \sigma_{s}^{2}\right)}
$$

where, $P\left(X \mid H_{1}, h, \sigma_{s}^{2}\right)$ and $P\left(X \mid H_{0}, \sigma_{s}^{2}\right)$ represents likelihood functions under $\mathrm{H}_{1}$ and $\mathrm{H}_{0}$ respectively as,

$$
\begin{gathered}
P\left(X \mid H_{0}, \sigma_{s}^{2}\right)=\prod_{n-0}^{N-1} \frac{1}{\pi^{M \sigma_{s}^{2 M}}} \exp \left[-\frac{X^{H}(n) X(n)}{\sigma_{s}^{2}}\right] \\
P\left(X \mid H_{0}, \sigma_{s}^{2}\right)=\prod_{n-0}^{N-1} \frac{1}{\pi^{M\left|h^{H}+\sigma_{s}^{2}\right|}} x \exp \left[\left(-X^{H}(n)\left(h h^{H}+\sigma_{s}^{2} I\right)\right)^{-1} X(n)\right]
\end{gathered}
$$

On the basis of the sensing results each CR user ma intains a channel history database as,

$$
\begin{aligned}
& \Phi_{s}^{k}=\left[\Phi_{s}^{1}, \Phi_{s}^{2}, \Phi_{s}^{3}, \ldots, \Phi_{s}^{k}\right] \\
& \text { where, } \quad \Phi_{s}^{k} \text { is }
\end{aligned}
$$

PU vectors show a particular channel during time slot $\mathrm{s}$ and $\mathrm{k}$.

$$
\Psi_{s}^{k}=\left[\Psi_{s}^{1}, \Psi_{s}^{2}, \Psi_{s}^{3}, \ldots, \Psi_{s}^{k}\right](5)
$$

where, $\Psi_{s}^{k}$ Specifies a specific channel history vector $\mathrm{k}$ for the time slot. The value of the $s$ is 100

$$
\mathrm{H}=\left\{\begin{array}{cccc}
\Psi_{1}^{1} & \Psi_{2}^{1} & \ldots & \Psi_{s}^{1} \\
\Psi_{1}^{2} & \Psi_{2}^{2} & \ldots & \Psi_{s}^{2} \\
\vdots & \vdots & \ddots & \vdots \\
\Psi_{1}^{k} & \Psi_{2}^{k} & \ldots & \Psi_{s}^{k}
\end{array}\right\}
$$

For the PUs on the $\mathrm{k}$ channels $\mathrm{H}$ denotes the background matrix. It preserves the history of each channel and based on this history the Transmission Possibility Scale (TPS) is determined for each available channel TPS suggests the upcoming usability of the channel. TPS is represented as.

$\eta_{s}^{k}=e^{-\left[\frac{\sum_{s-1}^{T_{s}} \Psi_{s}^{k}}{T_{s}}\right] \text { for } \Psi_{s}^{k}>}$

where $\eta$ represents TPS and $\Psi$ indicates the historical pattern of the PU on the channel T, number of time slots and it is calculated using (5). The summation value varies between 0 and 1 while the TPS varies between 0.3697 and 1 . The variables Ts and s can be used interchangeably, which are used to improve consistency. The TPS is designed to reduce breakdowns and overhead switches to increase QoS In CR the QoS criterion helps you to select the best source. Three separate operators such as selection, crossover, and mutation is used by the genetic algorithm directing people to an optimal global solution convergence. These initial assumptions are explicitly established as the binary strings of the particular variables, whereas an increasing number of Gas uses real encoding. The structure of the active subcarrier pattern distribution with reconfiguration parameter is based around DSM. After that, the receiver takes steps to make decisions to the new spectrum distribution of the present user.

Published By:

Blue Eyes Intelligence Engineering DOI: 10.35940/ijeat.C6083.049420

Journal Website: www.ijeat.org
\& Sciences Publication

102 (c) Copyright: All rights reserved. 
A new TPS gene has been inserted in chromosomes to facilit ate better QoS data rates, delay service times and reduced int erruptions (channel switching). Since collision with PU the TPS gene decreases the number of retransmission and consequently saves the transmission power. The key genes included in delivery are the frequency band (FB), power (PWR), modulation mechanism and (MOD) bit error rates (BERs), data ratio (DR) interferes with primary users (IWPU). Subsequently, the fitness assessment for each chromosome in the population is carried out in order to produce the chromosome population. The function fi is then determined by,

$$
f_{i}=\left\{\begin{array}{lc}
\left.\mid \frac{w_{i} \cdot\left|x_{i}-x_{i}^{d}\right|}{x_{i}^{d}}\right], & \text { if }\left|x_{i}-x_{i}^{d}\right|<x_{i}^{d} \\
w_{i}, & \text { otherwise. }
\end{array}\right.
$$

Chromosome fitness is provided by the use of "wi" that vari es with performance goal "fi" according to the QoS require ment.

$$
\begin{gathered}
F=\sum_{i=1}^{n} w_{i} \cdot f_{i} \\
=w_{d t} \cdot f_{d t}+w_{p w r} \cdot f_{p w T}+w_{b e r} \cdot f_{b e r}+w_{\text {ivpu }} \cdot f_{\text {iwp }}+w_{\text {IPS }} \cdot f_{\text {TPS }}
\end{gathered}
$$

The total fitness level is

$$
\text { Total fitness }(\%)=100\left[1-\sum_{i=1}^{n} w_{i} \cdot f_{i}\right]
$$

The probability of selection of the best population chromoso mes is

$$
P_{i}=\frac{f_{i}}{\sum_{i=1}^{n} f_{i}}
$$

The advancement of the two crossover points is then given. The mutation of the new generation is done and the mutation is used to change the single bits ' values in individual strings randomly. The string is added to the original population behind the selection, crossover and mutation, in order to add one population to the new population generation counter. The CR adaptive parameters are symbolized as $=(, \ldots, \mathrm{xr})$. The customizable parameters include FB, PWR, MOD, BER, IWPU, DR and TPS. F = $(\mathrm{f} 1, \mathrm{f} 2, \mathrm{f} 3, \ldots \mathrm{fq})$, where $\mathrm{q}$ corresponds to the number of objective functions. By changing the multi-target function to the single objective function via the relationship, the progression of generalization is achieved. Objective function formulation is given as,

$$
\begin{gathered}
f_{D R}=f(x)= \begin{cases}\frac{d_{r}-d_{c}}{d_{r}}, & d_{r}<d_{c} \\
1, & \text { otherwise }\end{cases} \\
f_{P T R}=1-\frac{P}{P_{\max }} \\
f_{B E R}=f(x)= \begin{cases}\frac{\log _{10}\left(\bar{P}_{b e}\right)}{\log _{10}\left(\bar{P}_{\operatorname{minbor}}\right)}, & P_{b e}<10^{-3} \\
1, & \text { otherwise }\end{cases} \\
f_{\text {IITPU }}=1-\frac{I}{I_{\max }} \\
f_{T P S}=\frac{\eta}{\eta_{\max }}
\end{gathered}
$$

Where $d_{r}$, represents data rate and $d_{c}$ indicates the data rate available on the channel. P, shows the full transfer power for the CR user on a given channel and Pmax. The minimum BER value is the average BER expressed by minber. Imax , Specifies a maximum limit on PU activity. The maximum value of a chance index for the given channel is $\eta$ a full potential for the CR. The average power factor $(\mu)$ is the power available to transmit the data in a given time frame.

\section{EXPERIMENTAL RESULTS AND ANALYSIS}

The proposed arrangement is intended to achieve a various performance objective through the Proportional Resource Algorithm and Genetic Algorithm, as a resolution in Cognitive Radio Networks on behalf of sub-carrier allocation and power allocation complexity for Cognitive Radio consumers.In this suggested scheme, CRN sub-carrier allocation is considered, wherever each CR has correct sensing and history management capabilities. The findings tracked are accurate in more general situations. The GA parameter settings are used for the DR set to $265 \mathrm{kbps}$, the PWR is $15 \mathrm{db}$ and the BER is $10-5$. In order to prove the performance of the GA is represented by means of RF parameters or genes. The simulations are repetitively performed meant for 100 times by way of the 200 GAs iterations to appraise the efficacy of the proposed scheme and output consisted of final optimized gene values. As a final point the finest chromosome is selected to inspect the performance of the objective function. Random channel selection systems follow the results of the suggested approach for the CR network.

QoS Requirement: FB $=400 \mathrm{MHz}, \mathrm{MOD}=\mathrm{QPSK}$, $\mathrm{BER}=11-9, \mathrm{SWR}=3 \mathrm{db}, \mathrm{IWPU}=0.25$, TPS $=0.9394$. Specifications: The results obtained are shown in figures subsequently. GA population settings $=12$, convergence $=$ 0.5 , mutations $=3$ percent, chromosomes $=32$, elits $=2$ percent and generation numbers $=100$. GA is a populationsized scale. The 100 iterations are conducted to determine the best TPS solution for its optimum value and thus provide the required level of DR and the above-mentioned goals. In this procedure, the average objective function value to validate the efficacy of the proposed scheme is determined using four different modes. The diverse objectives are addressed for different applications and situations in each mode.

The objective in High Throughput (HT) mode is to maximize average throughput for CR users. This mode is suited to applications with higher data rates. The power response mode (PR) is suitable for power- For various applications such as emergencies the BER mode helps to lower the BER.sensitive applications and for CR networks and text messages. The QoS mode helps to decrease the number of PU interrupts or channel switches. This mode also has power savings due to the collision with the PU, by avoiding retransmission. Assumption is made that the fitness function depends equally on all selective Rayleigh frequency channels. Figure 1 demonstrates the algorithm development as a generation function with and without a mutation. 


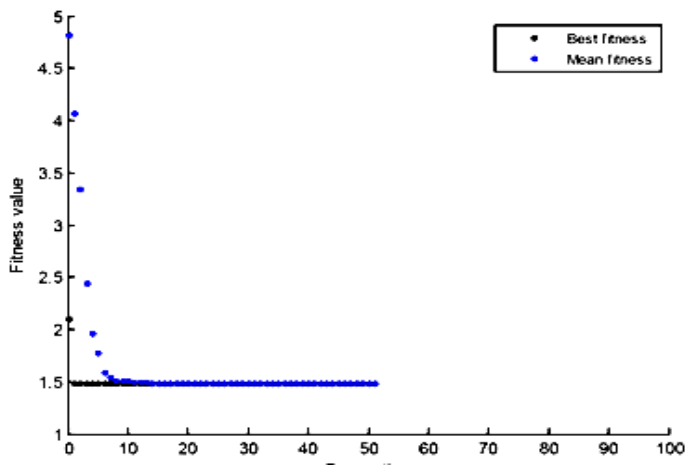

Figure 1. Fitness Value Vs Number of Generations

That bit in each new child string which throws a random number between 0 and 1 , when that number is less than $1 / 64$ and flips the bit. The best fit individual is shown in Figure 2 . Simulated for 100 iterations and above are shown. The tests are shown for the first 60. Fitness and mean fitness average shown in Figure 1 against its generations. The Best fitness is in the range of 1.4881 and the Mean fitness is in the range of 1.4882 .

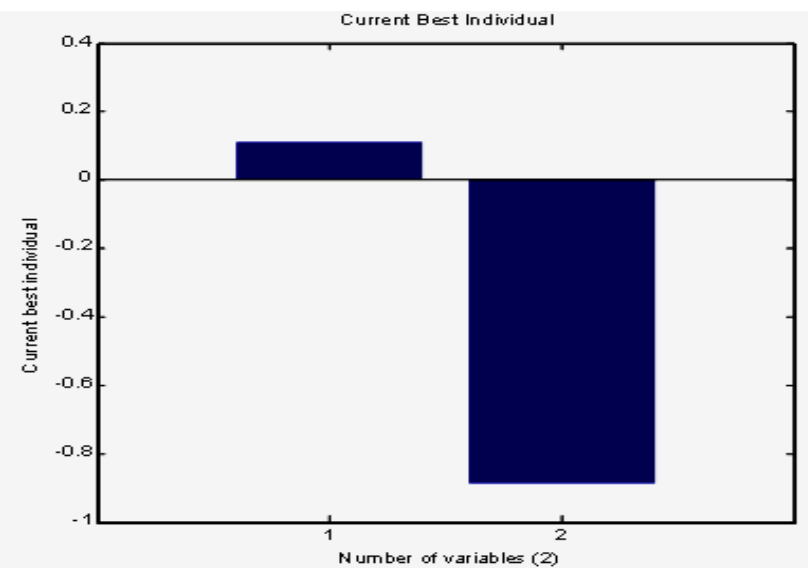

Figure 2. Best fitted individual Vs Number of variables.

Figure 2 shows the number of best person Vs current variables. The first variable defines the projected data rate and the second is the channel date rate. The optimum individual's fitness is 1 hence after the first iteration values of the individuals shown in the 0 to 0.8 range.

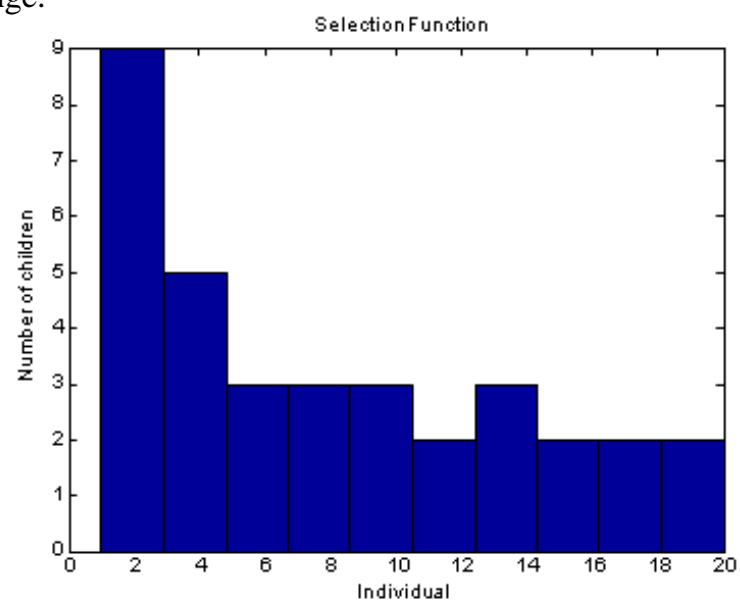

Figure 3. Number of Children Vs Number ofindividual.
Figure 3 shows every individual population of children. Average distance between individuals is shown in Figure 4.

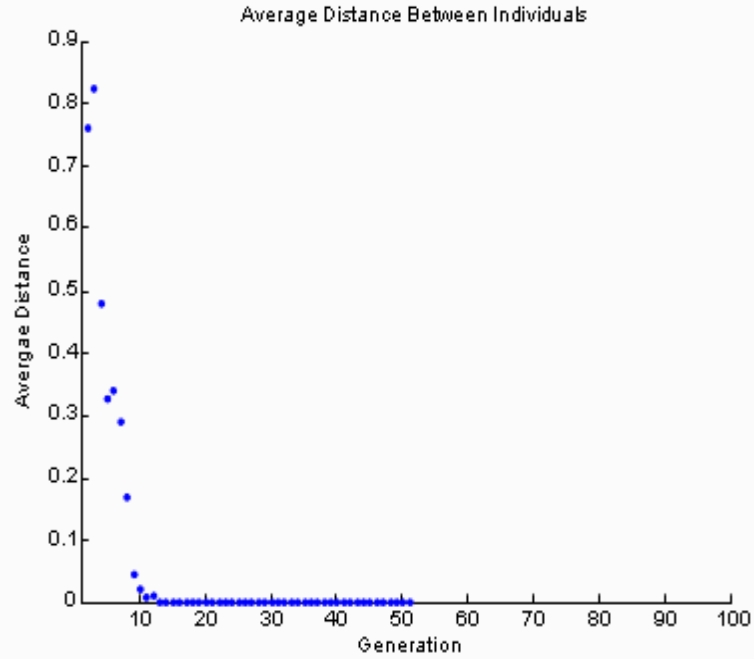

Figure 4. Average Distance VsNumber of Generations

It tests the distance from parent to child and shows that the optimal value of 0 determines the objective function solution. Best: Mean: 1.4881 1.482. Table 1 summarizes the structure of the genes being considered for implementation of GA.

Table1: Gene Structure

\begin{tabular}{|l|c|c|c|}
\hline Gene & $\begin{array}{c}\text { Bits } \\
\text { required }\end{array}$ & \multicolumn{1}{c|}{ Range } & Levels \\
\hline FB & 7 & $54-862 \mathrm{MHz}$ & 100 \\
\hline MOD & 2 & $\begin{array}{l}\text { BPSK,QPSK,8-QAM,16- } \\
\text { QAM }\end{array}$ & 4 \\
\hline BER & 4 & $10-1-10-16$ & 16 \\
\hline DR & 5 & $50-1.65 \mathrm{Mbps}$ & 32 \\
\hline PWR & 4 & $3-48 \mathrm{dBm}$ & 16 \\
\hline IWPU & 4 & $0.0625-1$ & 16 \\
\hline TPS & 4 & $0.3679-0.9394$ & 16 \\
\hline
\end{tabular}

The parameters and weights have been chosen appropriately which depend on the QoS. Simulation experiment performed for the device mentioned where the total of subcarrier numbers is 64 varying with different times. The observed outcomes related with The different modes of operation and the value of the relevant gene are presented in Figure 5, Figure 6, Figure 7 and Figure 8.

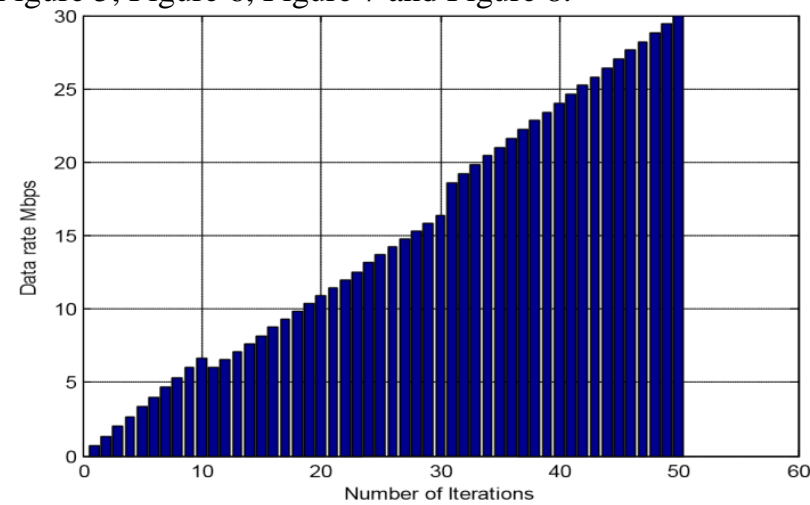

Figure 5. Data Rate in DR Mode

Published By:

Blue Eyes Intelligence Engineering \& Sciences Publication

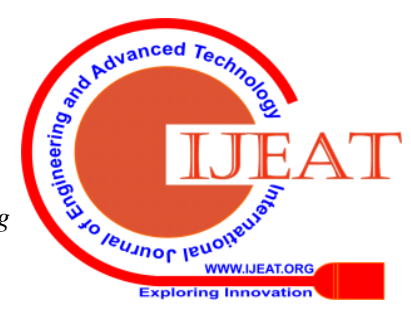


The high-performance mode weights $65 \%$. In this mode, the objective data rate function is close to 51 iterations of optimum value. The target feature of power reduction is maximum weight in Power Responsive mode. Within a few iterations, the power needed in this mode is reduced. In this case the transmission potential scale gene reaches a $65 \%$ of its weight, which after 25 iterations reaches its maximum value and the rest of its parameters achieve its own optimum values as well.

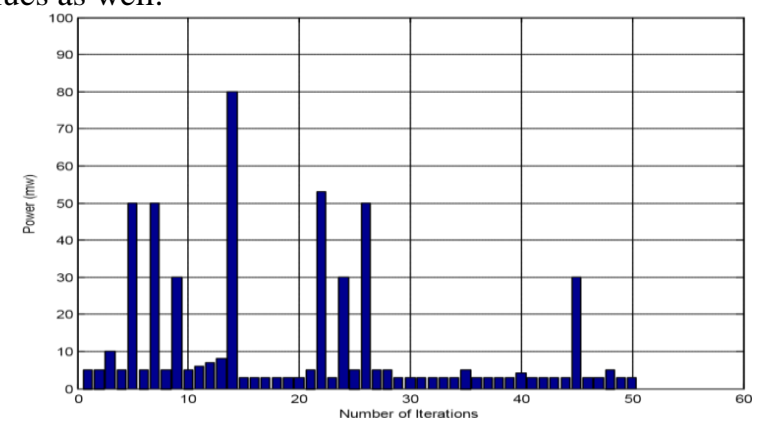

Figure 6. Power in PR Mode

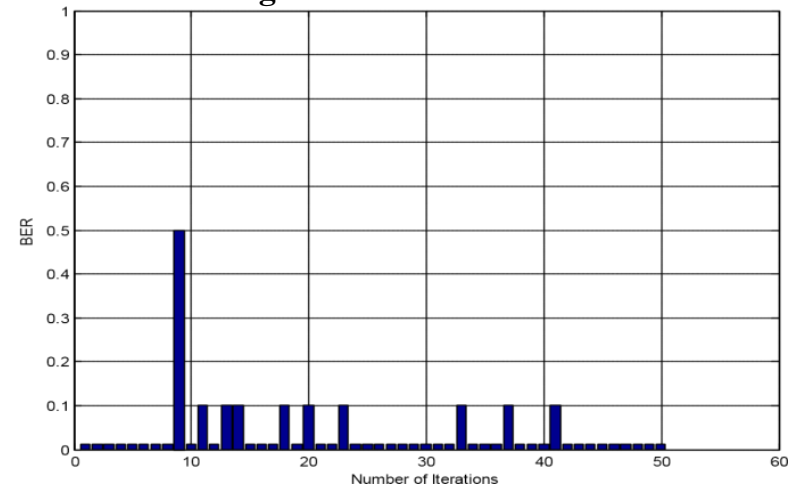

Figure 7. BER in BER Mode

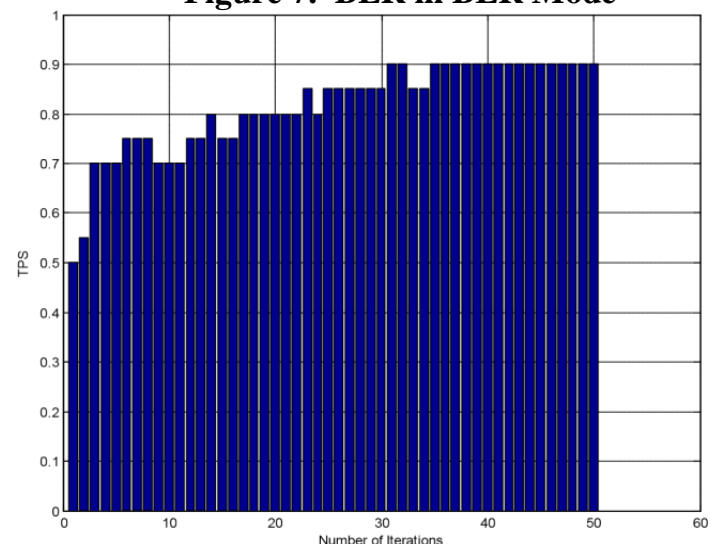

Figure 8. TPS in QoS Mode

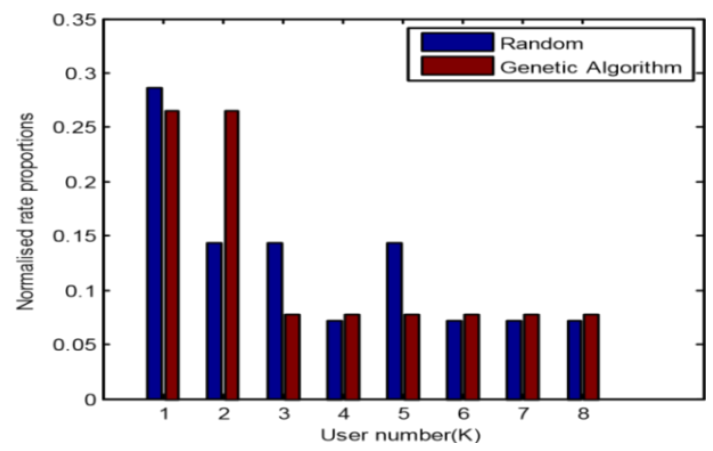

Figure 9.Number of UsersVs Rate Proportional

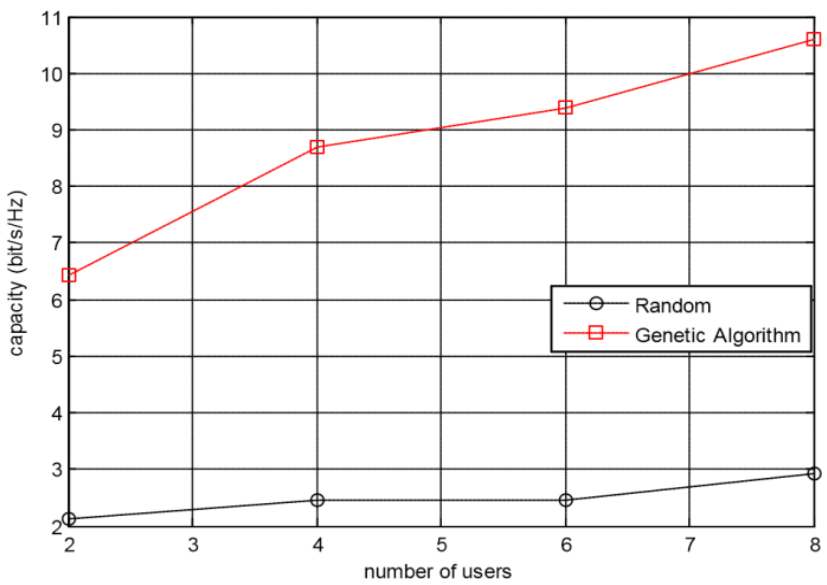

Figure 10. Channel capacity Vs Number of Users

Figure 9 gives an idea of the users with the best service attempt only. The capacity of each Secondary User shall be measured in proportion to the different speed. The results show that the genetic algorithm guarantees the ability to adjust operating parameters for a different secondary user. The results of the proposed subsidiary allocation system over the number of users are shown in Figure 10. The suggested scheme discusses the optimal solution, including channel information and number of users. The simulation results confirm the information is being fed directly and thus the sub-carrier connections plays an important role in enhancing system performance. Genetic Algorithm becomes attractive as the number of users increases and has been compared with random scheme. In addition to that considerably reduces the computational complexity and achieves higher capacities.

\section{CONCLUSION}

The proposed subcarrier allocation and power allocation scheme for cognitive radio network is designed via genetic algorithm to achieve different performance objectives as solutions. The proposed scheme is established its efficacy through allocating maximum data rate for all subcarrier. Besides that it is used to maximize the total network throughput which is to maximize the number of satisfied users under the bandwidth constraints where the services are guaranteed in terms of quality requirements from a dynamic bandwidth management perspective. The suggested scheme covers all major problems, such as channel characteristics, maintaining QoS without interfering with Primary User's operations.

To select the optimal channel for Cognitive Radio networks and channel accessibility where users are designed to interact without primary users. The findings obtained by using the genetic algorithm used for genetic operations and then by Cognitive radio users are tailored to the respective sub-contractor. The objective function is played a vital role on different transmission modes to yield better results. The solutions observed confirms the fitness function of the individual parameters which increases with an increase in the number of generations and reach the optimal solution within the pool of solutions available.

All parameters that are independent to one another and that are considered for fitness purpose.

Blue Eyes Intelligence Engineering

\& Sciences Publication

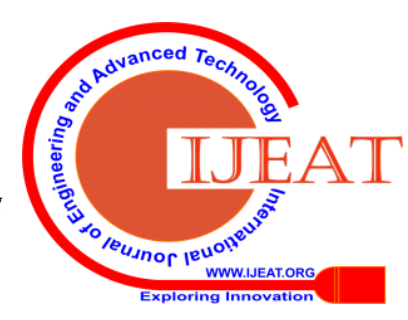


The key effects of the proposal are seen in reducing the number of channels and switches, as well as increasing the efficiency and data rate of the networks. The benefit makes the Active subcarrier Pattern for Reconfiguration scheme which is suitable for power sensitive applications like cognitive radio network.

\section{REFERENCES}

1. Mitola, J. and Maguire, G.Q., "Cognitive Radio: Making Software Radios More Personal”, IEEE Personal Communications Magazine, Volume 6, issues 4, Pages 6-13, 1999, https:// doi:10.1109/98.788210.

2. Farah.J. and Marx.F, Combining Strategies for the Optimization of Resource Allocation in a Wireless Multiuser OFDM System. International Journal on Electronics Communication, Volume 61 Issue 10, Pages 665-677, November 2007. https:// doi: 10.1016/j.aeue.2007.01.002

3. Akyildiz.I.F., "Cooperative Spectrum Sensing in Cognitive Radio Networks: $\quad$ A Survey", Physical Communication, Volume 4 issue 1, Pages40-62, March 2011, https://doi.org/10.1016/j.phycom.2010.12.003

4. Qin.H., Su.J. and Du.Y., "Multiobjective Evolutionary Optimization Algorithm for Cognitive Radio Networks", IEEE IEEC, Ternopil, 1617 May 2009, Pages 164-168, IEEE Xplore: 28 July 2009, https:// doi: 10.1109/IEEC.2009.39

5. Hegazy, Tarek, "Optimization of Resource Allocation and Leveling Using Genetic Algorithms", Journal of Construction Engineering and Management.Volume 125, issue 3, Pages 167-175, June 1999, https://doi:10.1061/(ASCE)0733-9364(1999)125:3(167)

6. Wang.P., Fang.J., Hang.N. and Lee.H., "Multiantenna-Assisted Spectrum Sensing for Cognitive Radio", IEEE Transactions on Vehicular Technology, Volume 59, Issue 4, Pages 1791-1800, May 2010, https:// doi: 10.1109/TVT.2009.2037912

7. Thomas W. Rondeau, Bin Le, Christian J. Rieser, Charles W. Bostian, "Cognitive Radios with Genetic Algorithms: Intelligent Control of Software Defined Radios", Proceeding of the SDR 04 Technical Conference and Product Exposition, Pages 1-6, 2004.

8. Guo-ying Yang and Hong Jiang, "Parameters Optimization of Cognitive Radio Based on DNA Genetic Algorithm", Fuzzy Information and Engineering Volume 2, Pages 809-816, 2009, https://doi.org/10.1007/978-3-642-03664-4 88

9. Saleem Aslam, Kyung Geun Lee, "CSPA:Channel Selection and Parameter Adaptation Scheme Based on Genetic Algorithm for Cognitive Radio Ad Hoc networks", EURASIP Journal on Wireless Communications and Networking, Volume 349, Pages 1-15, November 2012, https://doi.org/10.1186/1687-1499-2012-349

10. Jamal Elhachmi and Zouhair Guennoun, "Cognitive Radio Spectrum Allocation Using Genetic Algorithm", EURASIP Journal on Wireless Communications and Networking, Pages 1-11, May 2016, https://doi.org/10.1186/s13638-016-0620-6

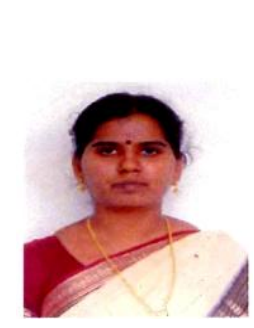

\section{AUTHOR PROFILE}

Rajalakshmi presently a full time Research Scholar, Department of Computer Science,Bharathidasan University,Tiruchirappalli.She has completed MCA,M.Phil Degree in Computer Science and pursuing his full time Ph.D in Optimization of Spectrum Allocation in Cognitive Radio Technology. Previously, she has worked as Lecturer at Department of Computer Science, Dhanalakshmi Srinivasan College of Art \& Science for Women, Perambalur from 2002 to 2009. Then she worked as Assistant Professor at Department of Computer Science, Rover Engineering College, Elambalur from 2009 to 2013.

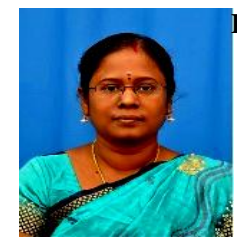

P. Sumathy is currently Assistant Professor at Department of Computer Science, Bharathidasan University, Tiruchirappalli. She has completed M.Sc in Computer Science, M. Phil in Computer Science and Ph.D in the area of Image Retrieval. She has around 12 years of Academic Experience and 9 years of Research Experience. She specializes in Content based Image Retrieval, Multimedia Information Retrieval and Data Mining. She has published several Research papers in National and International Journals of repute. 